\title{
A FIXED POINT THEOREM OF MARKOV-KAKUTANI TYPE FOR A COMMUTING FAMILY OF CONVEX MULTIVALUED MAPS
}

\author{
XIONGPING DAI \\ Department of Mathematics, Nanjing University \\ Nanjing 210093, People's Republic of China \\ E-mail: xpdai@nju.edu.cn
}

\begin{abstract}
Let $\Gamma$ be a commuting family of upper semicontinuous convex multivalued maps of $\boldsymbol{K}$ into itself with nonempty closed values, where $\boldsymbol{K}$ is a nonempty compact convex subset of a locally convex Hausdorff topological vector space $\boldsymbol{E}$. We then show that the Markov-Kakutani fixed-point theorem holds; that is, there exists at least one point $\boldsymbol{x} \in \boldsymbol{K}$ such that $\boldsymbol{x} \in u(\boldsymbol{x})$ for all $u$ in $\Gamma$.
\end{abstract}

Key Words and Phrases: fixed-point theorem, multimaps, invariant measure of multimaps, locally convex Hausdorff topological space.

2010 Mathematics Subject Classification: 47H10, 54H25, 54C60.

Acknowledgments. The author wishes to thank the anonymous referee for her/his helpful comments and some corrections for the earlier version of this paper.

This work was supported partly by National Natural Science Foundation of China grant \#11431012, 11271183 and PAPD of Jiangsu Higher Education Institutions.

\section{REFERENCES}

[1] R.P. Agarwal, M. Balaj, D. O'Regan, Common fixed point theorems and minimax inequalities in locally convex Hausdorff topological vector spaces, Appl. Anal., 88(2009), 1691-1699.

[2] C.D. Aliprantis, K.C. Border, Infinite Dimensional Analysis, 2nd ed., A Hitchhiker's Guide, Springer-Verlag, Berlin Heidelberg NY, 1999.

[3] J.-P. Aubin, H. Frankowska, Set-Valued Analysis, Birkhäuser, Boston Basel Berlin, 1990.

[4] J.-P. Aubin, H. Frankowska, A. Lasota, Poincaré's recurrence theorem for set-valued dynamical systems, Ann. Polon. Math., 54(1991), 85-91.

[5] M. Balaj, A common fixed point theorem with applications to vector equilibrium problems, Appl. Math. Lett., 23(2010), 241-245.

[6] M.M. Day, Fixed point theorems for compact convex sets, Illinois J. Math., 5(1961), 585-590.

[7] R.E. Edwards, Functional Analysis: Theory and Applications, Dover Publications, New York, 1995.

[8] Ky Fan, Fixed point and minimax theorems in locally convex topological linear spaces, Proc. Nat. Acad. Sci. U.S., 38(1952), 121-126.

[9] I. Glicksberg, A further generalization of Kakutani fixed point theorem with applications to Nash equilibrium points, Proc. Amer. Math. Soc., 3(1952), 170-174.

[10] S. Kakutani, Two fixed-point theorems concerning bicompact convex sets, Proc. Imp. Acad. Tokyo, 14(1938), 242-245. 
[11] S. Kakutani, A generalization of Brouwer's fixed point theorem, Duke Math. J., 8(1941), 457459.

[12] L.-J. Lin, C.-S. Chuang and Z.-T. Yu, Generalized KKM theorems and common fixed point theorems, Nonlinear Anal., 74(2011), 5591-5599.

[13] A. Markov, Quelques théorèmes sur les ensembles abéliens, Dokl. Akad. Nauk. SSSR (N.S.), 10(1936), 311-314.

[14] I. Namioka, Kakutani-type fixed point theorems: A survey, J. Fixed Point Theory Appl., 9(2011), $1-23$.

[15] S. Park, Ninety years of the Brouwer fixed point theorem, Vienam J. Math., 27(1999), 187-222.

[16] J. Schauder, Die fixpunktsatz in funktionalräumen, Studia Math., 2(1930), 171-180.

[17] A. Tychonoff, Ein fixpunktsatz, Math. Ann., 111(1935), 767-776.

Received: July 17, 2014; Accepted: December 4, 2014. 
